\title{
Vaccines work
}

Vaccination has successfully reduced the burden of infectious diseases worldwide, but stagnating immunization coverage and lack of effective vaccines for many endemic and newly emerging pathogens pose a threat to sustainable global health. In light of World Immunization Week 2018, which highlights the importance of high vaccination coverage, Nature Communications is taking stock of current advances and barriers in vaccine development and distribution.

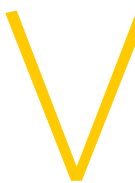

accination is one of the most successful and cost-effective public health interventions. The World Health Organization (WHO) estimates that an average of 2 to 3 million deaths are prevented every year, thanks to worldwide vaccinations. Many more lives are protected from acute disease and life-long disabilities caused by infections.

The international community and policymakers have long recognized the immense benefit of vaccination and have put a concerted effort and billions of dollars into global immunization campaigns; this has successfully reduced burden of infectious diseases. For example, in 1988, at the beginning of the Global Polio Eradication Initiative (GPEI), 1000 children worldwide were paralyzed every day due to poliomyelitis. Today, most countries are free of polio and only three countriesAfghanistan, Nigeria, and Pakistanremain endemic. A polio-free world is within reach.

Yet polio is only one of currently 25 vaccine-preventable infections ${ }^{1}$. Global immunization coverage is too low and an estimated 1.5 million deaths could be avoided by "simply" increasing the number of vaccinated children ${ }^{2}$.

One reason for stagnating or declining vaccination coverage in many regions of the world is a lack of resources. The Global Alliance for Vaccines and Immunizations (Gavi) was created in 2000 to address this problem and increase access to vaccines in lower-income countries. Gavi has contributed to vaccination of roughly 640 million children. It aims to strengthen health systems and improve sustainability of national immunization programs, with the ultimate goal of enabling developing countries to carry out vaccination independently. This approach has shown success and several of the initially included countries no longer depend on financial support from Gavi.

Another barrier is a societal resistance to vaccination, largely rooted in an unfounded mistrust and unreasonable fear from adverse side effects. Measles is a prime example. A safe vaccine has been available since the 1960s and has protected millions of children's lives worldwide. But vaccination coverage is currently too low to prevent outbreaks. In 2017, 14,451 measles cases were reported in Europe, a more than three-fold increase from $2016^{3}$. Measles infection routinely causes complications and approximately one of every 1000 measles infected children will die. In contrast to common misperception, this holds true in developed countries with state of the art healthcare. Italy for example reported roughly 4400 measles cases between January and August of 2017, nearly 2000 of which were hospitalizedthree children died $^{4}$.

This year's World Immunization Week with the theme "Protected Together, \#VaccinesWork" highlights the importance of immunization and encourages everyone, from donors to the general public, to help to increase vaccination coverage. We can all do our part by getting vaccinated.

Reaching high coverage for already approved vaccines is not the only challenge we are facing. We currently lack vaccines for many infectious diseases, including respiratory and diarrheal diseases, fungi, 
HIV, tuberculosis, malaria, and emerging pathogens. Vaccine development for many of these diseases has proven a formidable challenge, and previously successful approaches are unlikely to be sufficient.

There is cause for optimism. Technological advances have opened up promising new avenues in vaccine research and development, including antigen discovery and design. Antibodies isolated from protected individuals pinpoint to immunogenic areas in microbial molecules ${ }^{5}$. Structure-based antigen design stabilizes and exposes epitopes to guide the immune response to highly conserved domains ${ }^{6}$. Nanoparticles enhance stability and modify immunogenicity of selected molecules? mRNA vaccines serve as a new platform in clinical development that, in addition to other advantages, promise rapid, and inexpensive production ${ }^{8}$. This nonexhaustive list illustrates encouraging progress in design of next generation vaccines.

Rational antigen design and vaccine development crucially rely on an in depth understanding of the protective immune response. Yet animal models are often poor predictors of the human immune response to a particular pathogen, rendering specimens from naturally infected individuals invaluable. Each sample may be interrogated with a multitude of assays, including serology, transcriptomics, metabolomics, and others. Not surprisingly, systems vaccinology plays an increasingly important role to make sense of the wealth of data 9 . Identified correlates of protection may be used as a surrogate of vaccine efficacy during development and enable objective comparison of vaccine candidates.

Natural infection is not the only option to study the human immune response. Deliberate infection of volunteers under controlled conditions, so-called human challenge studies, represent an intriguing alternative, at least for some pathogens. While such experiments may have a checkered history, regulations, and ethical guidelines are in place today to ensure safety and proper utilization ${ }^{10}$.
Despite advances in research and development, vaccines for many emerging pathogens are unlikely to be developed, largely due to uncertainties in regulatory pathways and markets. This leaves us vulnerable to large-scale outbreaks of emerging viruses, such as Ebola and Zika, with devastating effects on health and tremendous socioeconomic costs. To address this, the Coalition for Epidemic Preparedness Innovations (CEPI) was founded in 2016. CEPI facilitates and finances vaccine development for high risk pathogens to the stage of a safe and immunogenic candidate, with the goal to enable rapid effectiveness trials in case of an epidemic.

There are already encouraging success stories of similar initiatives. The Meningitis Vaccine Project (MVP) was launched in 2001 to develop a vaccine protecting from yearly meningitis outbreaks in sub-Saharan Africa. It was clear that the vaccine would need to cost less than 50 US cents per dose to be affordable, which is about one-tenth of a typical vaccine. MVP succeeded with a hitherto unconventional approach-they developed the vaccine together with a manufacturer in India. This is the first vaccine specifically developed for Africa.

Vaccines are arguably the best approach to stop infectious diseases, and benefits can go beyond individual and public health gains ${ }^{11}$. Non-health outcomes include, for example, higher educational attainment ${ }^{12}$, which may affect a child's economic future. Increasing vaccination coverage and providing universal access to vaccines are crucial to realise sustainable global health and a fair chance of a healthy life for every child.

Published online: 24 April 2018

\section{References}

1. WHO. Global Vaccine Action Plan 2011-2020 http://www.who.int/immunization/ global_vaccine_action_plan/ GVAP_doc_2011_2020/en/.
2. WHO. Immunization coverage http://www.who. int/mediacentre/factsheets/fs378/en/.

3. European center for disease prevention and control. Monthly measles and rubella monitoring reports https://ecdc.europa.eu/en/measles/ surveillance-and-disease-data/monthly-measlesrubella-monitoring-reports.

4. Filia, A. et al. Ongoing outbreak with well over 4,000 measles cases in Italy from January to end August 2017-what is making elimination so difficult? Euro Surveill. 22, 30614 (2017).

5. Lopez-Sagaseta, J. et al. Crystal structure reveals vaccine elicited bactericidal human antibody targeting a conserved epitope on meningococcal fHbp. Nat. Commun. 9, 528 (2018).

6. Kupl, D. W. et al. Structure-based design of native-like HIV-1 envelope trimers to silence non-neutralizing epitopes and eliminate CD4 binding. Nat. Commun. 8, 1655 (2017).

7. Deng, L. et al. Double-layered protein nanoparticles induce broad protection against divergent influenza A viruses. Nat. Commun. 9, 359 (2018).

8. Pardi, N. et al. mRNA vaccines-a new era in vaccinology. Nat. Rev. Drug. Discov. 17, 261-279 (2018).

9. Fourati, S. et al. Pre-vaccination inflammation and B-cell signalling predict age-related hyporesponse to hepatitis B vaccination. Nat. Commun. 7, 10369 (2016).

10. WHO. Human challenge trials for vaccine development: regulatory considerations http:// www.who.int/biologicals/expert_committee/ WHO_TRS_1004_web_Annex_10.pdf?ua=1.

11. Wilder-Smith, A. et al. The public health value of vaccines beyond efficacy: methods, measures and outcomes. BMC Med. 15, 138 (2017).

12. Anekwe, T. D. et al. The causal effect of childhood measles vaccination on educational attainment: a mother fixed-effects study in rural South Africa. Vaccine 33, 5020-5026 (2015).

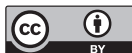

Open Access This article is licensed under a Creative Commons Attribution 4.0 International License, which permits use, sharing, adaptation, distribution and reproduction in any medium or format, as long as you give appropriate credit to the original author(s) and the source, provide a link to the Creative Commons license, and indicate if changes were made. The images or other third party material in this article are included in the article's Creative Commons license, unless indicated otherwise in a credit line to the material. If material is not included in the article's Creative Commons license and your intended use is not permitted by statutory regulation or exceeds the permitted use, you will need to obtain permission directly from the copyright holder. To view a copy of this license, visit http:// creativecommons.org/licenses/by/4.0/.

(c) Macmillan Publishers Ltd, Part of Springer Nature 2018 